\title{
固相浓度对深海采矿矿浆洜空化性能影响规律
}

\author{
徐海良 ${ }^{1,2}$ 徐 聪 ${ }^{1}$ 曾义聪 ${ }^{1}$ 吴 $\quad$ 波 $^{1,2}$
}

(1. 中南大学机电工程学院 长沙 410083;

2. 中南大学高性能复杂制造国家重点实验室 长沙 410083)

\begin{abstract}
摘要: 为分析固相浓度对深海采矿矿浆泵空化特性的影响, 通过空化核子理论、质能方程建立气相和液相、固相和液相之间 的联系, 探求气固两相之间的理论关系, 进行固相参数对深海采矿矿浆䈋空化性能影响分析, 并采用 mixture 多相流模型, RNG $k-\varepsilon$ 湍流模型, Schnerr and Sauer 空化模型, 在 fluent 软件中对矿浆泉进行稳态空化仿真。比较不同颗粒浓度对矿浆泵流 场压力分布、气相分布及工作性能的影响, 为矿浆原空化特性提供依据。研究结果表明: 空化发生时的临界气泡半径与固相 浓度及其流量可通过液体压强建立联系, 固相颗粒浓度越大、固相流量越大, 空化将提前发生, 抗空化性能将下降; 随着固 相浓度的增加, 在矿浆意首级叶轮叶片背面入口处压力降幅增大, 气相体积分数增大, 原扬程减小, 汽蚀余量减小。
\end{abstract} 关键词：深海采矿矿浆原；空化特性；固相浓度；理论分析；数值模拟 中图分类号: P744; TH313

\section{Effect of Solid-phase Concentration on Cavitation Performance of Deep-sea Mining Pump}

\author{
XU Hailiang ${ }^{1,2}$ XU Cong ${ }^{1}$ ZENG Yicong ${ }^{1} \quad$ WU Bo $^{1,2}$
}

(1. School of Mechanical and Electrical Engineering, Central South University, Changsha 410083;

2. State Key Laboratory of High Performance Complex Manufacturing, Changsha 410083)

\begin{abstract}
In order to analyze the influence of solid concentration on the cavitation characteristics of deep-sea mining pump, the relationship between gas phase and liquid phase, solid phase and liquid phase are established through the theory of cavitation nucleus and the energy equation. And the effect of solid-phase parameters on the cavitation performance of deep-sea mining pump is studied. The cavitation steady simulation of slurry pump is carried out by fluent software using the mixture multiphase flow model, RNG $k-\varepsilon$ turbulence model and Singhal fully cavitated model. The influence of different particle concentration on the pressure distribution, gas distribution and working performance of the slurry pump are given, which provides the theoretical basis for the cavitation characteristics of the slurry pump. The results show that the critical bubble radius and the solid concentration and the flow rate can be established by the liquid pressure when the cavitation occurs. The larger the solid phase particle concentration is, the larger the solid phase flow will occur. The anti-cavitation performance of pump will decrease. With the increase of solid concentration, the pressure decreases at the inlet of the impeller blade of the primary pump, as well as the volume fraction of the gas and the anti-cavitation performance.
\end{abstract}

Key words: deep sea mining pump; cavitation; solid-phase concentration; theoretical analysis; numerical simulation

\section{0 前言}

陆地资源日渐减少，使得深海采矿技术越发重 要。目前, 德国、美国、日本、韩国等国家都已经 完成了深海采矿的海上试验, 各发达国家都在积极 推进海洋资源的商业化开采 ${ }^{[1]}$ 。各国对深海采矿系

* 国家自然科学基金资助项目(51775561)和湖南省自然科学基金资助项目, 深海采矿阀控式清水百水力提升设备工作机理及设计方法(2018JJ2522)。 20180520 收到初稿, 20181120 收到修改稿
统进行深入研究, 普遍认为由集矿系统, 扬矿系统, 水面支持系统以及测控系统组成的深海采矿装备最 具商业化开采前景。扬矿系统作为重要的部分其作 用是将矿石从海底输送至海面, 矿浆泵性能好坏直 接影响扬矿系统的输送效率。国内外学者对深海采 矿矿浆洜的结构和泵内固液两相流流场进行了大量 的研究, 德国 KSB 公司在 20 世纪 70 年代对输运 粗颗粒提升泵的水力模型、流道等进行研究, 采 用具有离心泵和轴流洜双重特性的混流洜作为提

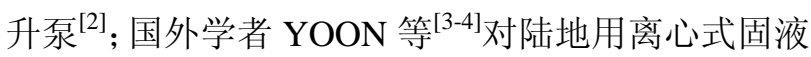


洜进行了性能试验和数值模拟, 结果表明该固液矿 浆泵难以达到水力管道提升系统的工作要求。我国 中南大学杨放琼等 ${ }^{[5]}$ 根据垂直管道水力输送理论, 提出深海采矿矿浆泵设计理论公式; 陈奇 ${ }^{[6]}$ 在此基 础上, 构建了矿浆洜三维模型, 对矿浆泵内的固液 两相流进行数值模拟研究。现今对该矿浆洜空化方 面的研究很少。空化作为水力旋转机械中常见的现 象, 对泵的运转具有重要影响。其发生往往会影响 洜内流体的正常流动、形成噪声、振动、空蚀破坏, 改变泵外特性参数(如轴功率、扬程、流量), 严重 时甚至会使整个系统停止工作 ${ }^{[7]}$, 这对深海采矿系 统而言十分严重。目前对于各种类型的泵大都有相 关的空化特性研究, 例如李文广 ${ }^{[8]}$ 采用全空化模型 计算离心泵的“扬程-汽蚀余量”曲线, 虽然趋势与试 验结果一致, 但误差较大。赵宇等 ${ }^{[9]}$ 采用 FBM 湍流 模型和 Zwart 空化模型对一型单级轴流洜和一型串 列原空化特性进行数值分析, 计算得出的空化特性 曲线与试验结果吻合良好。深海采矿矿浆泵输送的 是固液两相流，发生空化时将形成复杂的三相流。 本文首次考虑了固相对洜空化性能的影响, 从理论 和数值模拟上进行多相相互作用的分析研究, 进一 步揭示深海采矿矿浆䈋的空化特性。

\section{1 固相浓度对矿浆泵空化特性影响 理论分析}

选取在二级矿浆百首级叶轮级上的 1,2 两个截 面如图 1 所示, 假设 1 处为泵初始入口处, 2 为临 界空化发生处, 分析从 1 到 2 这一过程中固液气之 间关系。

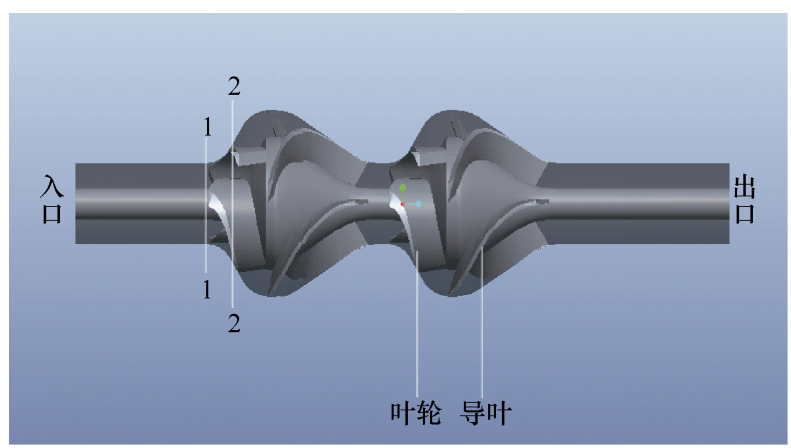

图 1 深海采矿矿浆泵三维模型

\section{1 气相与液相相关关系}

根据空化核子理论, 在某一温度下流体中某处 的局部压力低于该液体的饱和蒸气压使得液体中的 气核爆发性生长, 气泡破裂产生高压就会对洜体结 构形成冲击, 持续的作用使得泵的性能越来越低。
1947 年, Hervey 提出边壁表面缝隙存气模 式 ${ }^{[10]}$ 。设平衡状态的孤立球形空泡内存在水蒸气 和其他少量气体, 忽略气体扩散, 空泡静力平衡条 件为

$$
p=p_{v}+p_{g}-\frac{2 \delta}{r_{b}}
$$

式中, $p$ 为液体压强, $r_{b}$ 为空泡半径, $p_{v}$ 为空泡中 蒸汽的饱和蒸汽压强, $\delta$ 为液体表面张力系数, $p_{g}$ 为空泡中气体组分压强。

空泡内气体压强 $p_{g}$ 的变化可以按理想气体状 态方程和等温过程变化。

按理想气体状态方程变化

$$
p_{g}=\frac{N T}{r_{b}^{3}}
$$

式中, $N$ 为气体常数, $T$ 为绝对温度。

按理想气体等温过程变化

$$
p_{g}=p_{g_{0}}\left(\frac{r_{b_{0}}}{r_{b}}\right)^{3}
$$

式中, $p_{g_{0}}$ 为初始状态空泡内气体压强, $r_{b_{0}}$ 为空泡 初始半径。

对应上述两种变化过程, 可以得到式(1)的两种 表达式

按理想气体状态方程变化

$$
p=p_{v}+\frac{N T}{r_{b}^{3}}-\frac{2 \delta}{r_{b}}
$$

按理想气体等温过程变化

$$
p=p_{v}+p_{g_{0}}\left(\frac{r_{b_{0}}}{r_{b}}\right)^{3}-\frac{2 \delta}{r_{b}}
$$

对深海采矿矿浆百从 1 到 2 过程进行分析, 1 处的液体压强为 $p_{1}, 2$ 处的液体压强为 $p_{2}$, 根据上 述公式，按照理想气体状态方程变化有

$$
\begin{aligned}
& p_{1}=p_{v}+\frac{N T_{1}}{r_{1}^{3}}-\frac{2 \delta}{r_{1}} \\
& p_{2}=p_{v}+\frac{N T_{2}}{r_{2}^{3}}-\frac{2 \delta}{r_{2}}
\end{aligned}
$$

式中, $r_{1} 、 r_{2}$ 分别为 1 和 2 处的气泡半径。

$$
\begin{gathered}
N=\left(p_{1}-p_{v}+\frac{2 \delta}{r_{1}}\right) \frac{r_{1}^{3}}{T_{1}} \\
p_{2}=p_{v}+\left(p_{1}-p_{v}+\frac{2 \delta}{r_{1}}\right) \frac{T_{2}}{T_{1}}\left(\frac{r_{1}}{r_{2}}\right)^{3}-\frac{2 \delta}{r_{2}}
\end{gathered}
$$

由 1 到 2 的过程视为等温变化, 即 $T_{1}=T_{2}$, 式 (9)可以写为 


$$
p_{2}=p_{v}+\left(p_{1}-p_{v}+\frac{2 \delta}{r_{1}}\right)\left(\frac{r_{1}}{r_{2}}\right)^{3}-\frac{2 \delta}{r_{2}}
$$

在矿浆泵断面 2 处为气泡破灭的临界状态, 此 时压强 $p_{2}$ 为临界压强, $r_{2}$ 为气泡临界半径, 对于式 (10), 当 $\frac{\mathrm{d} p_{2}}{\mathrm{~d} r_{2}}=0$ 时, 可求出该临界状态值。所以对 式(10)求微分如下

$$
\frac{\mathrm{d} p_{2}}{\mathrm{~d} r_{2}}=\left(p_{0}-p_{v}+\frac{2 \delta}{r_{1}}\right) \frac{-3 r_{1}^{3}}{r_{2}^{4}}+\frac{2 \delta}{r_{2}^{2}}=0
$$

解得

$$
r_{2}=\sqrt{3\left(p_{1}-p_{v}+\frac{2 \delta}{r_{1}}\right) \frac{r_{1}^{3}}{2 \delta}}
$$

联立(10)、(12)得

临界半径

$$
r_{2}=-\frac{4 \delta}{3\left(p_{1}-p_{v}\right)}
$$

临界压强

$$
p_{2}=p_{v}-\frac{4 \delta}{3 r_{2}}
$$

气相和液相之间的联系通过液体压强和气泡半 径建立, 当液体压强为 $p_{2}$ 时, 处于汽蚀临界点, 此 时气泡半径为 $r_{2}$ 。

\section{2 固相与液相相关关系}

在固液两相流体中存在下述关系

$$
Q_{m} \rho_{m}=Q_{s} \rho_{s}+Q_{w} \rho_{w}
$$

式中, $Q_{m} 、 Q_{s} 、 Q_{w}$ 分别为浆体流量、固体流量、 水流量; $\rho_{m} 、 \rho_{s} 、 \rho_{w}$ 分别为浆体密度、固体密度、 水密度。

流体从 1 到临界汽蚀发生 2 处, 根据质能方程, 对于该过程可以列出

$$
\left(p_{1}-p_{2}\right) Q_{m}=\rho_{s} Q_{s} \frac{V_{2 s}^{2}-V_{1}^{2}}{2}+\rho_{w} Q_{w} \frac{V_{2}^{2}-V_{1}^{2}}{2}
$$

式中, $v_{1} 、 v_{2} 、 v_{2 s}$ 分别为 1 处的液体速度、 2 处的 液体速度、 2 处的固体速度。

对于式(16)两边同除以 $Q_{m} \rho_{m} g$, 可以得到

$$
\frac{p_{1}-p_{2}}{\rho_{m} g}=\frac{v_{2}^{2}-v_{1}^{2}}{2 g}\left[1+\frac{Q_{s} \rho_{s}}{Q_{m} \rho_{m}}\left(\frac{v_{2 s}^{2}-v_{1}^{2}}{v_{2}^{2}-v_{1}^{2}}-1\right)\right]
$$

固相和液相之间的联系通过式(17)中的相关参 数建立, 可以看出固相颗粒浓度和固相流量体积对 过程 1 到 2 的压强变化有影响。

\section{3 固相与气相相关关系}

第 $2 、 3$ 节中通过气泡半径和液体压强的关系以 及固相浓度流量和液体压强的关系分别建立了气相
和液相、固相和液相之间的联系, 以压强为中间量, 进行固相与气相之间联系的建立, 将式(14)代入式 (17)得

$$
\frac{p_{1}-p_{v}+\frac{4 \delta}{3 r_{2}}}{\rho_{m} g}=\frac{v_{2}^{2}-v_{1}^{2}}{2 g}\left[1+\frac{Q_{s} \rho_{s}}{Q_{m} \rho_{m}}\left(\frac{v_{2 s}^{2}-v_{1}^{2}}{v_{2}^{2}-v_{1}^{2}}-1\right)\right]
$$

气泡破灭是因为外界压力与饱和蒸汽压相等, 即 $p_{1}=p_{v}$, 式(18)即为

$$
\frac{4 \delta}{3 r_{2} \rho_{m} g}=\frac{v_{2}^{2}-v_{1}^{2}}{2 g}\left[1+\frac{Q_{s} \rho_{s}}{Q_{m} \rho_{m}}\left(\frac{v_{2 s}^{2}-v_{1}^{2}}{v_{2}^{2}-v_{1}^{2}}-1\right)\right]
$$

依据式(19)可以看出气泡破灭临界半径与固相 颗粒密度和固相流量有关系。其他参数不变时, 随 着固相颗粒密度 $\rho_{s}$ 和固相流量 $Q_{s}$ 的增大, 气泡破灭 的临界半径 $r_{2}$ 将减小, 意味着空化发生将提前, 泵 的抗汽蚀性能变差。

2 不同固相浓度对矿浆葲空化特性的 仿真分析

分析计算采用的深海采矿二级矿浆葲设计结构 如图 2 所示, 主要设计参数: 设计流量 $Q_{v}=800 \mathrm{~m}^{3} / \mathrm{h}$ (工作流量 $420 \mathrm{~m}^{3} / \mathrm{h}$ ), 扬程 $H=35 \mathrm{~m}$, 转速 $n=1450$ $\mathrm{r} / \mathrm{min}$, 汽蚀余量 $\mathrm{NPSH}_{a}=5 \mathrm{~m}$ 。泵入口直径 $D_{0}=240$ $\mathrm{mm}$, 叶轮吸入口直径 $D_{1}=244 \mathrm{~mm}$, 叶轮外径 $D_{2}=401.7 \mathrm{~mm}$, 叶轮出口宽度 $b_{2}=54 \mathrm{~mm}$, 叶轮叶片 数 $Z_{1}=3$, 导叶叶片数 $Z_{2}=4$ 。

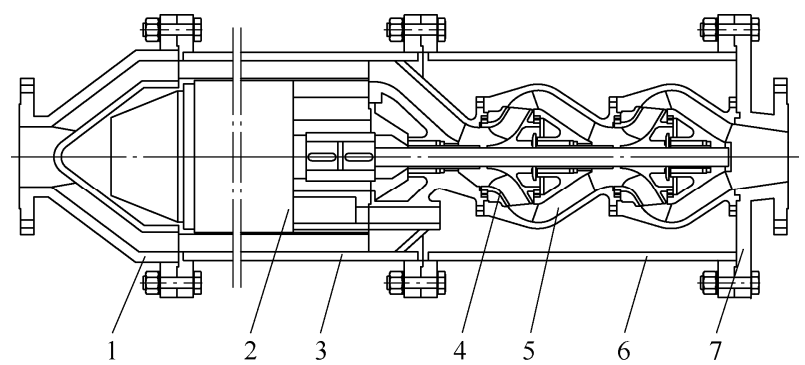

图 2 矿浆泵结构图

1. 进口法兰 2. 潜水电机 3. 电机筒体 4. 叶轮 5. 空间导叶 6. 泵筒体 7. 出口法兰

深海采矿矿浆百输送的是含有锰结核的海水 浆体, 发生空化时形成复杂的固液气三相流。海 水物理性质与其地理位置分布相关, 根据海水平 均盐浓度 $3.5 \%$, 参考《氯碱工业理化常数手册》 中浓度为 $3.5 \%$ 的氯化钠溶液的物理性质。海水中 压强温度因素的特殊性, 气相的性质参考《工程 常用物质的热物理性质手册》。各相物理性质如表 1 所示。 
表 1 物理参数

\begin{tabular}{lc}
\hline \multicolumn{1}{c}{ 物理性质 } & 数值 \\
\hline 海水密度 $/\left(\mathrm{kg} \cdot \mathrm{m}^{-3}\right)$ & 1025 \\
海水黏度 $/(\mathrm{Pa} \cdot \mathrm{S})$ & $1.673 \times 10^{-3}$ \\
海水饱和蒸气压 $/ \mathrm{Pa}$ & 806.45 \\
空气密度 $/\left(\mathrm{kg} \cdot \mathrm{m}^{-3}\right)$ & 0.01927 \\
空气黏度 $/(\mathrm{Pa} \cdot \mathrm{S})$ & $8.8 \times 10^{-6}$ \\
镍结核密度 $/\left(\mathrm{kg} \cdot \mathrm{m}^{-3}\right)$ & 2040 \\
锰结核平均粒径 $/ \mathrm{mm}$ & $20 \sim 50$ \\
锰结核浓度 $(\%)$ & $5 \sim 10$ \\
\hline
\end{tabular}

\section{1 基本假设}

为保证计算的可行性及结果的准确性, 做如下 假设: 固液两相流为连续的不可压缩流体, 物理特 性为常数, 第一相为海水, 第二相为锰结核颗粒, 第三相为空气, 结核颗粒和气泡假设为规则的球体。 考虑三相之间的曳力作用, 以及速度滑移, 第一相 和第三相之间发生质量交换存在相变。

\section{2 控制模型}

深海采矿矿浆百输送的浆体颗粒具有相同的性 质, 空化时为多相流流动, 相间存在速度差, 同时 为了更好地收玫, 故选择 mixture 模型, 该模型是 一种简化的多相流模型, 主要实现求解混合相的连 续性方程、能量方程、动量方程等。mixture 模型假 定离散固相为拟流体, 与连续液相具有相同的力学 特性, 拟流体的流动仍然采用宏观连续介质原理中 守恒方程进行描述 ${ }^{[11]}$ 。原内流体流动为湍流流动, 为了得到连续相和离散相比较真实的流动特征, 选 择 RNG $k-\varepsilon$ 湍流模型进行模拟 ${ }^{[12]}$ 。

Fluent 中提供了三种空化模型 Schnerr and Sauer 模型、Zwart-Gerber-Belamri 模型与 Singhal 模型, 前两者模型在数值计算方面略粗糙且收玫较 快, Singhal 模型考虑了未溶解气体作用, 但稳定性 较差。Schnerr and Sauer 模型与湍流模型有更好的 兼容性, 因此本文选择 Schnerr and Sauer 模型作为 空化模型进行仿真。

\section{3 网格划分}

在数值模拟矿浆洜流场之前, 需要离散化矿浆泉 流道的几何模型, 生成网格。计算网格包括非结构化 网格和结构化网格两类, 非结构化网格内部点不具有 相同的毗邻单元, 适用于复杂几何。结构化网格内部 节点具有相同的毗邻单元, 网格生成的速度快, 数据 结构简单网格生成的质量好, 与实际的模型更容易接 近。但结构化网格只适用于形状规则的几何; 叶轮与 空间导叶空间扭曲程度高, 几何形状极其复杂, 叶轮 及空间导叶流道更适合非结构网格。

在叶轮流道进口处和空间导叶流道出口处各增
加一段出水管来提高模拟的准确性。运用 ICEM CFD 前处理软件进行网格划分, 在进水管与叶轮流 道进口之间、叶轮流道出口与空间导叶流道进口之 间、空间导叶流道出口处和出水管之间引入 interface 交界面, 对静网格与动网格进行动静耦合(图 3)。

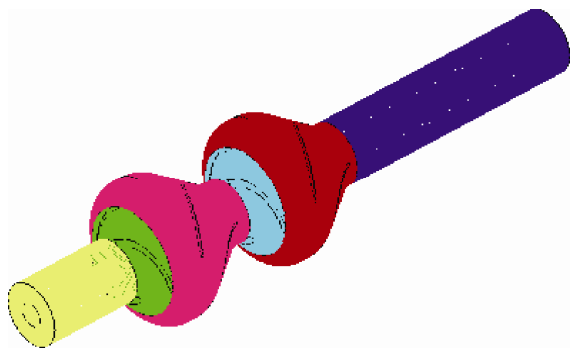

图 3 全流道技术网格

\section{4 仿真分析}

在工作流量 $Q_{v}=420 \mathrm{~m}^{3} / \mathrm{h}$, 颗粒粒径 $d=20 \mathrm{~mm}$, 转速 $n=1450 \mathrm{r} / \mathrm{min}$ 工况下, 依据中国大洋协会研究 报告《大洋多金属结核中试采矿系统 $1000 \mathrm{~m}$ 海上 试验总体系统技术设计》, 该采矿系统的输送矿石浓 度技术指标为 5\% 10\%, 采用等差法, 分别取颗粒 体积浓度 $C_{v}$ 为 $4 \% 、 6 \% 、 8 \% 、 10 \%$ 的固液两相流场 进行全流道空化数值仿真。分析矿浆洜全流道静压， 入口静压和出口静压分别达到-200 kPa、800 kPa, 首级叶轮处压力比第二级叶轮处压力低的多, 如图 4 所示。选择矿浆洜导叶和叶轮单独分析, 静压力 云图如图 5 所示, 压力在首级叶轮叶片背部入口处 最低, 相比于其他部分更容易发生空化现象, 因此 本文对首级叶轮及选取的叶轮最大轴截面进行压 力、气相分布的分析, 并计算泵的必须汽蚀余量 $\mathrm{NPSH}_{a}$ 和扬程 $H$, 分析不同颗粒体积浓度对矿浆洜 空化特性的影响。
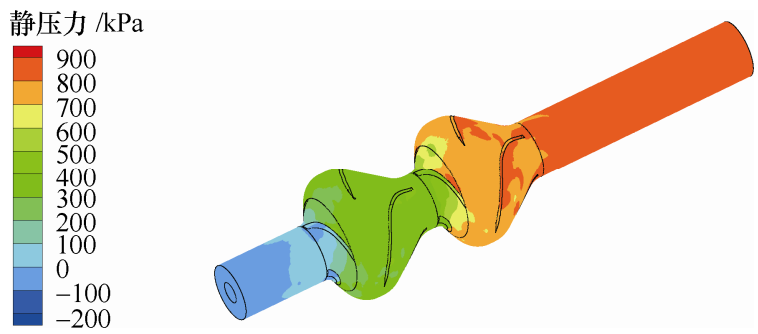

图 4 全流道静压力云图

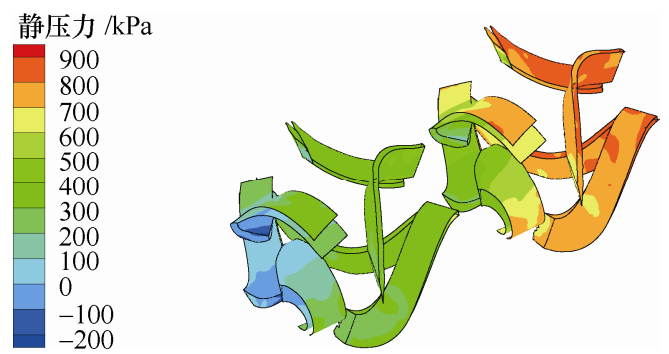

图 5 叶轮导叶静压力云图 


\subsection{1 固体颗粒体积浓度对矿浆葲内压力及气相} 分布的影响

图 6 为深海采矿矿浆泵首级叶轮叶片在不同固 相浓度的工况下的静压分布, 由该图可以看出压力 随着叶片吸力面和压力面的流线方向逐渐增加, 压 力最低点出现在叶片背面入口处, 形成一定的低压 区，易发生空化现象。随着结核颗粒浓度的增加， 低压区区域扩散, 当结核颗粒浓度为 $4 \%$ 时, 只在 叶片背面入口处有一小部分低压区, 当浓度增大到 $10 \%$ 时, 叶片背面入口处的低压区域增大, 且与低 浓度相比出现了更低的压力区域, 是由于在泵中当 水流速度下降时, 颗粒在惯性作用下速度大于水流, 两者间形成速度差, 这使得固相颗粒某处压力下降, 颗粒浓度越大, 单位体积内固相数量越多, 低压区 域更广泛, 为空化的发展提供了条件。
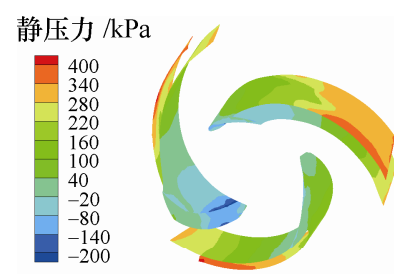

$C=4 \%$

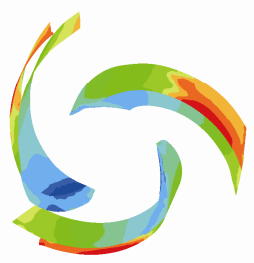

$C=8 \%$

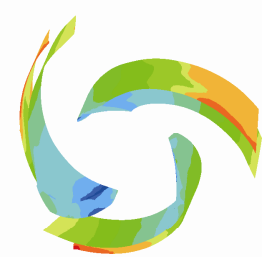

$C=6 \%$

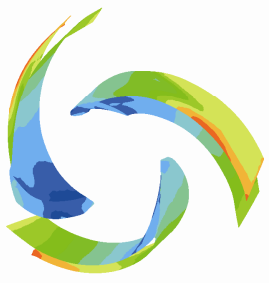

$C=10 \%$
图 6 不同颗粒浓度叶轮静压力云图

对选取的叶轮最大轴截面进行处理, 绘制其静 压力等值线图 7 , 从图 7 可以看出最低压力分布在
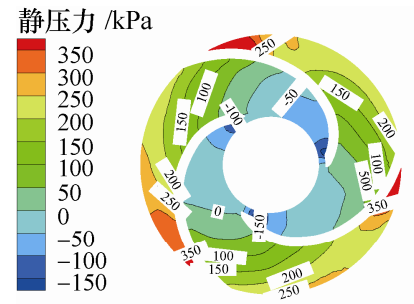

$C=4 \%$

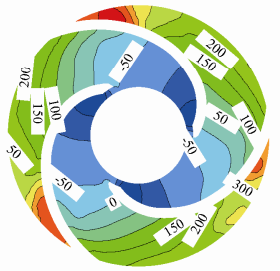

$C=8 \%$

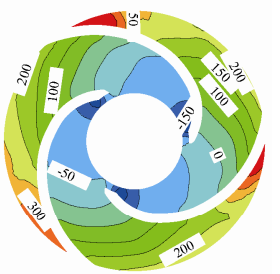

$C=6 \%$

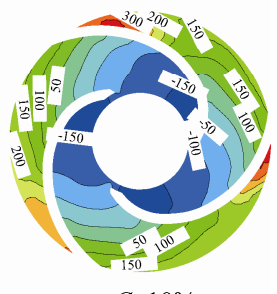

$C=10 \%$
图 7 不同颗粒浓度叶轮轴截面静压力等值线
叶片背部入口处达到-150 kPa, 最高压力分布在叶 片正面出口处达到 $350 \mathrm{kPa}$ 。结核颗粒浓度从 4\%增 加到 $10 \%$ 的过程中，明显的有低压区域面积增大的 现象，表明气相的分布区域扩散。

图 8 为首级叶轮在不同浓度的结核颗粒工况下发 生空化时的气相体积分布, 由该图可以看出, 气相主 要分布在叶轮叶片背面入口处，处在低压区，气泡随 着流线方向逐渐消失, 与压力变化趋势一致, 说明压 力的降低是空化发生的必要条件。当结核颗粒浓度为 $4 \%$ 时, 气相体积分数基本低于 0.1 , 但是分布的范围较 广。随着颗粒浓度的增加, 气相体积分数迅速增大, 当浓度增加到 $10 \%$ 时, 在叶轮叶片背面入口处气相体 积分数小区域达到 0.9 左右, 这对于叶轮将造成极大的 损害。从图中还能看出，气泡的分布区域并没有像低 压区那样连续成片分布，随着颗粒浓度的增加，气相 的分布更为分散, 主要原因是固相颗粒增多对气泡群 有冲击作用，使得气泡不能连续出现。选取的叶轮轴 截面上的气相体积分数分布也具有相似的规律(图 9)。

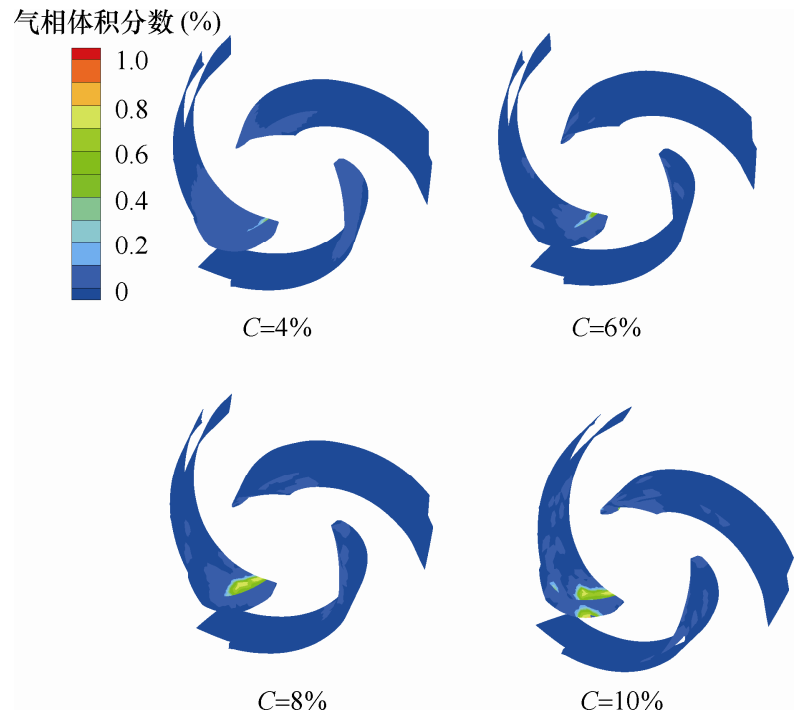

图 8 不同颗粒浓度气相体积分布

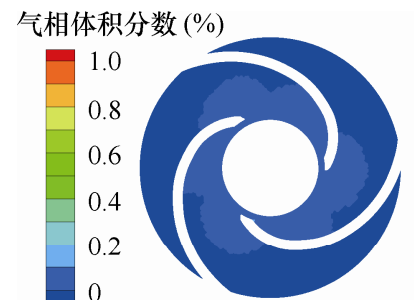

$C=4 \%$

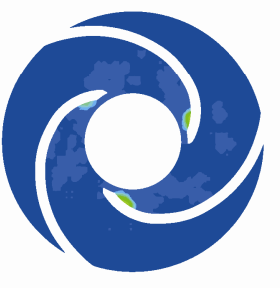

$C=8 \%$

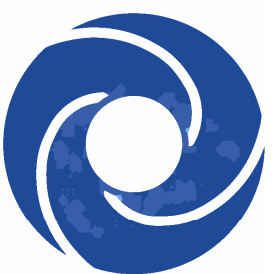

$C=6 \%$

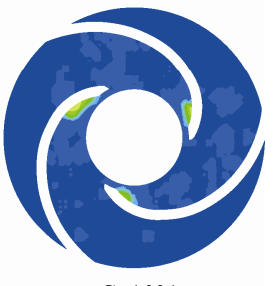

$C=10 \%$
图 9 不同颗粒浓度叶轮轴截面气相体积分布 
为了更直观地看到气相体积分数的变化, 对叶 轮最大轴截面进行处理, 在 tecplot360 后处理软件 中导出轴截面气相体积分数数据, 该数据共有 1970 个值点, 每个数值代表截面上各点的气相体 积分数, 所有点的气相体积分数的和与总个数的比 值就是气相占总体的比值, 可以通过该值分析气相 的量, 见图 10。从图 10 可以直接看出, 随固相浓 度的增加气相体积分数迅速增大, 在 $10 \%$ 浓度时达 到 0.8 左右, 是 $4 \%$ 浓度的 8 倍多, 颗粒浓度对空 化作用显著。

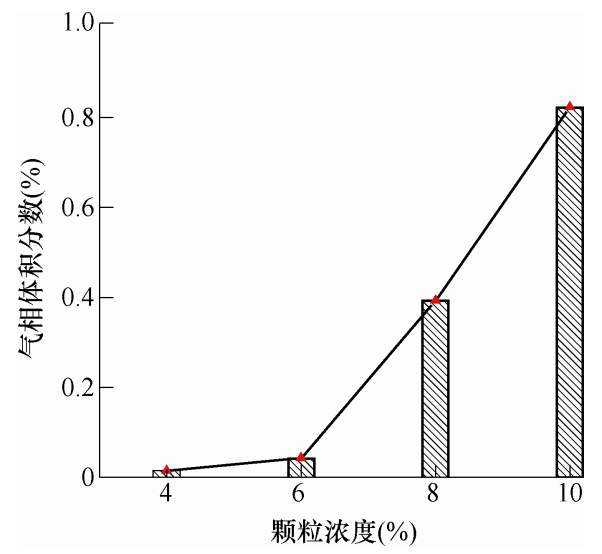

图 10 不同颗粒浓度叶轮轴截面气相体积分数

\subsection{2 矿浆洜扬程及必须汽蚀余量计算分析}

葲汽蚀余量 $\mathrm{NPSH}_{a}$, 是规定百要达到的汽蚀性 能参数, 其值越大说明泵的抗汽蚀性能越好。

$$
\mathrm{NPSH}_{a}=\frac{P_{i n}-P_{v}}{\rho g}
$$

式中, $P_{i n}$ 为矿浆泵进口总压, $P_{v}$ 为海水饱和蒸汽压, $\rho$ 为浆体密度。

矿浆百的扬程

$$
H=\frac{P_{o u t}-P_{i n}}{\rho g}+\Delta Z
$$

式中, $P_{\text {out }}$ 为矿浆百出口总压, $\Delta Z$ 为垂直方向进出 口截距差。

根据上述公式分别得到不同颗粒浓度下矿浆原 的扬程和汽蚀余量, 绘制曲线图如图 11 所示。

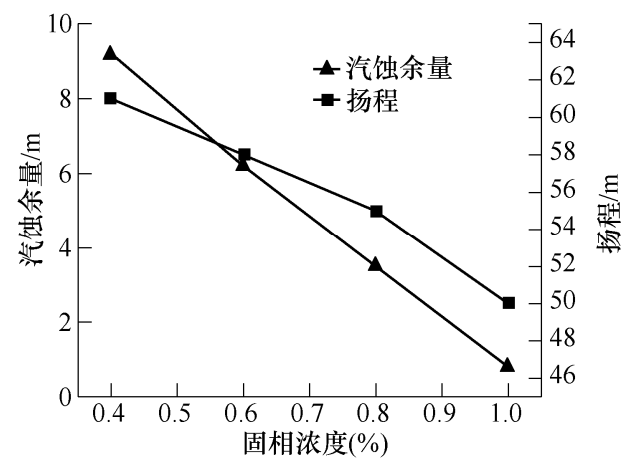

图 11 汽蚀余量扬程曲线图
固体颗粒浓度在 4\%时, 矿浆泵的扬程达到 61 $\mathrm{m}$, 汽蚀余量为 $9.2 \mathrm{~m}$, 此时矿浆洜抗汽蚀性能良好, 随着固相颗粒浓度的增加, 扬程逐渐下降, 汽蚀余 量逐渐上升，当固相浓度达到 $10 \%$ 时，扬程下降到 $50 \mathrm{~m}$, 汽蚀余量下降至最低 $0.8 \mathrm{~m}$, 两者随固体浓度 的变化趋近于线性。

\section{3 结论}

通过理论计算、数值仿真对深海采矿矿浆洜空 化特性进行研究, 探究不同固相颗粒浓度对矿浆空 化的影响, 得出以下主要结论。

(1) 通过液相压强可以建立空化发生时气相和 固相之间的联系, 气泡临界半径与固相浓度及其流 量之间存在负相关关系, 即固相颗粒浓度越大、固 相流量越大, 空化发生的气泡临界半径越小, 空化 将提前发生。

（2）深海采矿矿浆百空化发生的主要位置在首 级叶轮叶片背面入口处, 该区域流场压力低, 是空 化形成的必要条件。随着流线方向, 压力逐渐升高, 气泡消失。

（3）颗粒浓度对空化作用显著，固相浓度从 $4 \%$ 增大到 10\%的过程中, 矿浆洜空化现象逐渐明显, 首级叶轮叶片背面入口处气相体积分数急剧增大, 最大达到 0.9 ; 矿浆泵 $H$ 下降, NPSH 增大, 泵的 抗空化性能减弱, 与理论分析结果相吻合; 固相颗 粒的作用, 使气相分布不连续, 空化的作用范围进 一步扩大。

本文揭示了矿浆百空化特性的一些基本规律, 对于控制优化矿浆洜工作环境以及多相流仿真方面 具有一定的指导作用。除了颗粒浓度, 还有流量、 颗粒粒径、泵结构参数等都对矿浆洜空化特性具有 影响作用。由于矿浆洜结构复杂, 网格质量、模拟 条件都对计算结果有影响, 要准确全面地了解矿浆 洜空化特性, 后续研究可以利用现有技术如高速摄 像等进行试验上的验证。

\section{参 考 文 献}

[1] 董冰洁. 我国海洋多金属矿产资源研究现状及战略性 开发前景[J]. 世界有色金属，2016，12(1): 168-169.

DONG Bingjie. Research status and strategic development prospect of marine polymetallic mineral resources in China[J]. World Nonferrous Metals, 2016, 12(1): 168-169.

[2] 刘少军, 刘畅, 戴瑜. 深海采矿装备研发的现状与进展 [J]. 机械工程学报, 2014，50(2): 8-18.

LIU Shaojun, LIU Chang, DAI Yu. Status and progress on 
researches and developments of deep ocean mining equipments[J]. Journal of Mechanical Engineering, 2014, 50(2): $8-18$

[3] YOON C H, PARK Y C, LEE D K, et al. Hydraulic pumping test system of kigam for deep-sea manganese nodules[C]//Proceedings of 33rd Underwater Mining Institute, 2003: 131-135.

[4] H DING, F C VISSER, Y JIANG, et al. Demonstration and validation of a 3D CFD simulation tool predicting pump performance and cavitation for industrial applications[J]. Journal of Fluids Engineering, 2011, 133(1): 011101.

[5] 杨放琼, 陈奇, 曾义聪, 等. 深海采矿矿浆洜的设计方 法研究[J]. 合肥工业大学学报, 2014, 12(37): 1413-1418. YANG Fangqiong, CHEN Qi, ZENG Yicong, et al. Research on design method of slurry pump for deep-sea mining[J]. Journal of HeFei University of Technology. 2014, 12(37): 1413-1418.

[6] 陈奇. 深海采矿矿浆洜内固液两相流数值模拟及性能 预测研究[D]. 长沙: 中南大学, 2014.

CHEN Qi. Numerical simulation on solid-liquid two-phase flow and performance prediction of slurry pump for deep-sea mining[D]. Changsha: Central South University, 2014.

[7] 姬凯. 轴流洜叶轮内空化流动实验研究与数值计算[D]. 镇江: 江苏大学, 2010.

JI Kai. Experimental study and numerical calculation of cavitating flow in impeller of axial flow pump[D]. Zhenjiang: Jiangsu University, 2010.

[8] 李文广. 全空化模型预测离心泵汽蚀性能的准确度 [J]. 水洜技术，2013(5)：1-7.
LI Wenguang. Prediction of cavitation performance of centrifugal pump by full cavitation model[J]. Water Pump Technology, 2013(5): 1-7.

[9] 赵宇, 王国玉, 吴钦, 等. 基于计算流体力学的串列轴 流洜空化性能分析[J]. 机械工程学报，2014，50(6): 171-176.

ZHAO Yu, WANG Guoyu, WU Qin, et al. Analysis of cavitation performances of an axial flow tandem pump based on computational fluid dynamics[J]. Journal of Mechanical Engineering, 2014，50(6): 171-176.

[10］柯乃普 R T, 戴利 J W, 哈密脱 F G. 空化与空蚀 $[\mathrm{M}]$. 北 京: 水利出版社, 1981.

KNAPP $\mathrm{R} \mathrm{T}$, DAILY $\mathrm{J} \mathrm{W}$, HAMMITT $\mathrm{F} \mathrm{G}$. Cavitation[M]. Beijing: Water Conservancy Press, 1981.

[11] LAUNDER B E, SPALDING D B. The numerical computation of turbulent flows[J]. Computer methods in applied Mechanics and Engineering, 1974, 3(2): 269-289.

[12] COLOMBO M , FAIRWEATHER M. Multiphase turbulence in bubbly flows : RANS simulations[J]. International Journal of Multiphase Flow, 2015, 77: 222-243.

[13] YIN Fanglong, NIE Songlin, XIAO Shuhan, et al. Numerical and experimental study of cavitation performance in sea water hydraulic axial piston pump[J]. Proceedings of the Institution of Mechanical Engineers, 2016, 230(8): 716-735

作者简介: 徐海良, 男, 1963 年出生, 教授, 硕士研究生导师。主要从 事海洋采矿和矿山机械

E-mail: xuhailiang@csu.edu.cn

徐聪, 男, 1992 年出生。主要从事深海采矿研究。

E-mail: xu1992cong@163.com 